\title{
Deliberation as Genre: Mapping Argumentation through Relational Discourse Structure
}

\author{
Oier Imaz \\ oier.imaz@ehu.eus \\ University of the Basque Country \\ Vrije Universiteit Brussel \\ (UPV/EHU - VUB) \\ PRAXIS Research Group \\ Elhuyar plaza 2. \\ 20018 Donostia, Gipuzkoa
}

\author{
Mikel Iruskieta \\ mikel.iruskieta@ehu.eus \\ University of the Basque Country \\ (UPV/EHU) \\ IXA Group for NLP \\ Manuel Lardizabal pasealekua 1. \\ 20018 Donostia, Gipuzkoa
}

\begin{abstract}
Deliberation is an increasingly used concept in Argumentation Theory and Linguistic Analysis. But only recently research combined empirical and conceptual tool-boxes from these disciplines for the study of deliberative discourse. The aim of this article is to present a discursive analysis of deliberation as a genre using the relational discourse structure of texts. In particular, we want to see whether different features of deliberation genre map onto relational discourse structures of texts.To do so, authors analyze, in the framework of Rhetorical Structure Theory (RST), the relational discourse structure of a Basque-Spanish bilingual corpus of argumentative micro-texts written by citizens participating in a series of deliberative mini-publics. Results suggest that genre affects the relational discourse structures of texts and that we could analyze this effect in RST. Finally, we present, to our knowledge, the first annotated corpus-based genre analysis of the relational discourse structure of argumentative micro-texts (available online) with RST.
\end{abstract}

\section{Introduction}

Deliberation is an increasingly used concept in Argumentation Theory (Fairclough and Fairclough, 2013; van Eemeren, 2013). Argumentation Theory analyses deliberation as a genre, meaning discourse that is goal oriented, unfolds through stages and belongs to a discourse community (Bhatia, 2004). But only recently researches (Collins and Nerlich, 2015; Murray et al., 2013) focused on the linguistic analysis of deliberation as a genre.
The aim of this article is to present a discourse analysis of deliberation as a genre through the rhetorical structure of texts. We want to see whether the relational discourse structure of texts is influenced by different variables linking text to context: $i$ ) cultural variables (language) shaping the linguistic context of text (micro-context), ii) institutional design-choices (group composition) that feature the particular setting in which deliberation occurs (meso-context) and iii) stages of deliberation (macro-context). For Deliberative Democratic Theory and Argumentation Theory these three variables are relevant and affect the capacity of deliberation to achieve its purpose.

To do so, authors analyze the relational discourse structure of a Basque-Spanish bilingual corpus of argumentative micro-texts written by citizens participating in a series of deliberative mini-publics, in the framework of Rhetorical Structure Theory (RST) (Mann and Thompson, 1988).

Results suggest that the relational discourse structure of texts can be affected by the stage of discussion and design-choices, but not by the language of the participants.

Finally, we present, to our knowledge, the first annotated corpus-based genre analysis of the relational discourse structure of argumentative microtexts (available online ${ }^{1}$ ) with RST, in which discourse structures map onto deliberative dialogues. The time-cost of manual analysis in the RST framework limits the size of the corpus and the scope for application of this method. But advances have

\footnotetext{
${ }^{1}$ The corpus could be consulted at http://ixa2.si. ehu.es/diskurtsoa/rstfilo/
} 
been made for automatic discourse parsing of texts in Basque and Spanish (more info in Related Work Section).

\section{Theoretical Framework: Deliberation as an Argumentation Genre}

Argumentation is broadly defined here as the process through which people seek to reach conclusions through reasons (Fischer, 2012). Deliberation, on the other hand, is defined as a particular form of argumentation procedurally regulated by an ideal model, so that conclusions are reached only by the force of the better argument (Steiner, 2012). Both concepts testify for long and fruitful theoretical traditions: Argumentation Theory and Deliberative Democracy. Nevertheless, only recently they converged on the idea that deliberation is an argumentation 'genre' (Van Eemeren, 2016; Walton et al., 2014; Fairclough and Fairclough, 2013).

From the perspective of discourse studies, the key concept linking language and discourse is 'genre' (Miller, 1984). Genre entails that texts are goaloriented, involve stages and both influence linguistic choices. First, texts are goal-oriented because genre defines texts as communicative events geared to shared purposes in different discourse communities (Swales, 1990). Second, genre involves stages, because to accomplish shared purposes communicative events are structured in series of functional steps. Finally, Taboada (2004) suggests that genre is realized at the text level and it determines its structural organization.

In this paper, text analysis will follow basic guidelines from Taboada (2004, p. 29-36). According to her, genre analysis involves: $i$ ) finding a structural formula that will represent instances of a genre and ii) analyzing their linguistic characteristics ${ }^{2}$.

To complete the first task, we follow conventions from Argumentation Theory and, to complete the second task, we follow conventions from Rhetorical Structure Theory.

\footnotetext{
${ }^{2}$ It is specified step by step as follows: $i$ ) Identification of segments or series of segments; $i i)$ Definition of the social purpose; $i i i)$ Functional labeling of stages; $i v$ ) Specify obligatory and optional stages; $v$ ) Devise a structural formula; vi) Analyze the semantic and lexical-grammatical features for each stage (Taboada, 2004, p. 36)
}

\subsection{Deliberation Genre in Argumentation Theory}

Texts in the corpus are contributions written by citizens in a deliberative mini-public.

The institutional point or shared purpose of political deliberation is "preserving the democratic political culture by means of deliberation" (van Eemeren, 2013, p. 27). Indeed, organizers made this purpose explicit in The Konpondu Initiative. For example, the presentation leaflet underlines that it was motivated by the commitment of political representatives to build peace and contribute to political normalization. And it adds "the opinion of society, of people like you, is a fundamental contribution to that end".3 Moreover, the invitation letter ${ }^{4}$ established as rule 'mutual respect'; the central aspect of deliberation from the perspective of Argumentation Theory.

To accomplish this general purpose, the ideal template of deliberation defines seven different stages (McBurney et al., 2007, p.6). ${ }^{5}$ Nevertheless, this template should be adapted to real circumstances (Fairclough, 2016; Van Eemeren, 2016). In our case, texts collected inform over the Opening stage, or the question posed by the moderator. An InformPropose stage where citizen inform over their goals and perspectives and suggest possible courses of action. Next, a Consider stage where citizens consider a proposal placed by the moderator. To conclude, a Revise stage where proposals are accepted or rejected.

Finally, to advance towards the shared purpose citizens participating in a deliberative event are expected to fulfill a 'deliberative minimum' (Fairclough, 2016). In short, the ideal model of deliberative argumentation requires minimally "weighing reasons in favor of a claim against reasons against it (reasons supporting the counter-claim), or balancing each argument against a counter-argument" (Fair-

\footnotetext{
${ }^{3}$ Promotional leaflet of the Konpondu Initiative released by the Basque Government and collected in the course of this research.

${ }^{4}$ Invitation letter sent by Juan Karlos Goinetxea major of Bermeo to citizens, collected in the course of this research.

${ }^{5}$ Deliberation dialogue begins with an open question (Opening), followed by discussion on goals, constraints and perspectives (Inform), next proposals are placed (Propose), jointly considered (Consider), accepted or rejected (Revise) and an option is recommend (Recommend) before deliberation dialogue is closed (Conclude).
} 
clough and Fairclough, 2013, p. 50).

\subsection{Argumentative Discourse in RST}

Regarding the linguistic characteristics of deliberation genre, Taboada suggests that genre analysis should focus on variations as realized in the information structure (rhetorical relations), thematic structure (realization and progression) and cohesive structure (chains) of a text in its social and communicative context. Indeed, according to her, the relevant level of genre analysis is more textual than lexical-grammatical ${ }^{6}$ (Taboada, 2004, p. 2932). Our research focuses on one of those aspects in particular: the relational discourse structure. Coherence relations reflect the relational discourse structure of texts and could be analyzed with RST.

RST is an approach in which an analyst describes coherence between text fragments. To describe coherence, the analyst combines three main concepts: a) Elementary Discourse Units (EDU, henceforth) are independent or adverbial clauses. b) Recursive coherent relations between text fragments that have different effects on the reader: pragmatic or presentational relations and semantic or subject matter relations. As relations are recursive, a coherence relation can be a text fragment of other relation. c) Nuclearity, that is, the importance of a text fragment within the relation. Guided by the text, the analyst can describe which fragments are more important in the coherence relation (nucleus or satellite function) and also to other EDUs (central unit of a text).

Finally, we cluster coherence relations following Benamara and Taboada (2015) as Temporal, Thematic, Structural and Argumentative. The Argumentative Opposition subclass includes coherence relations that fulfill "the role of the classical thesis-antithesis structure." (Taboada and GómezGonzález, 2012, p.35) Therefore, we take the argumentative opposition subclass to represent the 'deliberative minimum' in the relational discourse structures of texts in our corpus.

\section{Methodology}

The annotation of the corpus follows basic guidelines of RST implemented in the "Multilingual Dis-

\footnotetext{
${ }^{6}$ On the contrary, lexical-grammatical features of texts and the realization of the three meta-functions in language are more closely related to register than they are to genre.
}

course TreeBank" (Iruskieta et al., 2015a). We will classify texts in regards to coherence relation classes conforming their relational discourse structure (Benamara and Taboada, 2015). Finally, we will statistically analyze whether different contextual variables (relevant for the genre of deliberation) affect the relational discourse structure of texts in our corpus.

\subsection{Corpus}

Texts in the corpus are contributions made by citizens in a deliberative exercise named The Konpondu Initiative $^{7}$. This initiative was held by the Basque Autonomous Government between 2007 and 2009 to foster citizens' participation in the resolution of the Basque conflict ${ }^{8}$. Texts are argumentative microtexts written by participants to assist their oral presentations. In short, they are fixed snapshots of different stages of the deliberative dialogue that took place in The Konpondu Initiative.

Mini-publics could be divided into two different phases depending on their structure. In the first phase, participants were called to express their opinion in response to a trigger question ${ }^{9}$ placed by the moderator, at the beginning of the exercise. Besides, participants were invited to reflect on the most interesting contributions made by others. In the second phase, the first question ${ }^{10}$ was more precise; it asked participants to underline different aspects (risks/opportunities/doubts). And, in addition to the concluding round, a direct question ${ }^{11}$ on the Consultation Law (Keating and Bray, 2006) was introduced in the middle of the exercise.

The complete set is composed of 2,850 plain texts ordered by language, group composition, stage of

\footnotetext{
${ }^{7}$ We want to thank Gorka Espiau and the Agirre Lehendakaria Center, Aitziber Blanco and Paul Rios from Lokarri, Jorge de la Herran from Agora and Igor Ahedo and Asier Blas from Parte-Hartuz (EHU) for helping us recollecting the documentation of The Konpondu Initiative.

${ }^{8}$ The initiative provided support for citizens participation via citizen fora held in 101 municipalities (162 fora), the diaspora (28 fora), the University ( 6 fora) and the youth council (6 fora), as well as a web fora (konpondu.net) where more than 20,000 comments were collected and over 1,000,000 hits documented

${ }^{9}$ In the current situation, what initiatives could contribute to a new opportunity for peace?

${ }^{10}$ In nowadays situation what problems and opportunities do you see to reach peace and political normalization?

${ }^{11}$ Do you agree that citizens be consulted to unlock the current situation?
} 


\begin{tabular}{|l|rrr|}
\hline Language & Texts & Relations & Words \\
\hline Basque & 100 & 1319 & 8900 \\
Spanish & 100 & 1205 & 11166 \\
\hline Total & $\mathbf{2 0 0}$ & $\mathbf{2 5 2 4}$ & $\mathbf{2 0 0 6 6}$ \\
\hline
\end{tabular}

Table 1: Corpus statistics

discussion, date, and town. As we show in Table 1, the corpus we present here is a selection of 200 texts using length as a general criterion, to capture those with denser discourse relational structures. We built a comparable set for both languages considering different stages of discussion and group-compositions.

\subsection{Annotation, Evaluation and Classification}

We annotated this Argumentative Basque-Spanish Treebank following the standard methodology in RST. We evaluated the reliability of the corpus following a two step process, first, implementing a qualitative evaluation method (Iruskieta et al., 2015a) and, second, by comparing RS-trees annotated by each annotator using the on-line freely available tool RSTeval (Maziero et al., 2009). ${ }^{12}$

Firstly, a novel annotator (A1) annotated some texts in both languages with the RSTTool (O'Donnell, 2000), following the standard way to annotate in RST: segmenting the text and, then, building the RS-tree modularly and incrementally (Pardo, 2005).

Secondly, a RST analyst (A2) annotated 20 texts (10 in Spanish and 10 in Basque) following the same methodology.

Thirdly, we compared RS-trees of A1 and A2 following a qualitative evaluation method proposed by (Iruskieta et al., 2015a) in two ways: $a$ ) with the extended set of RST relations and $b$ ) comparing a collapsed set of RST relations.

Fourthly, both annotators agreed on ideal templates or macro-structures on a case by cases basis. The annotation of the corpus was recomposed and, based on harmonized RS-trees, the annotation of relations was validated using RSTeval.

Fifthly, all the annotation data was automatically enriched morphosyntactically (lemmatized and POS-tagged) with Eustagger (Aduriz et al., 2003) for Basque and FreeLing (Carreras et al., 2004)

\footnotetext{
${ }^{12}$ RSTeval can be tested at http://www.nilc.icmc. usp.br/rsteval/.
}

for Spanish and it was exported to a database and showed in a friendly web-service environment using some tools developed in Iruskieta et al. (2015b).

Finally, coherence relations were clustered in classes following Benamara and Taboada (2015) and texts classified by their relational discourse structure and formatted for statistical analysis.

\subsubsection{Discourse Segmentation and Central Unit annotation}

Example (1) is a text selected from our Basque corpus (translations are ours), to explain the segmentation and central unit detection tasks. We segmented the text manually into Elementary Discourse Units (EDUs) following Iruskieta et al. (2015b). EDUs are independent sentences and adverbial clauses. The decision to segment manually was taken because texts were written by citizens; meaning they are full of grammatical mistakes (and a lot verbal ellipsis) and automatic segmentation will loose some EDUs (and therefore relations in subsequent phases) and produce more errors.

(1) [Espainiako alderdi nagusiek ez dute nahi ikusi geu geure artean ondo konpontzea. $]_{1}$ [Elkarrizketa edukitzerakoan, $]_{2}$ [norberaren "pretentsioak" apur bat bajatu behar dira, $]_{3}$ [akordio txikiak lortzeko, $]_{4} \quad$ [eta gero akordio handietara heltzeko. $]_{5}$ [Ondo dago herritarren artean foroak eta hitz egitea, $]_{6}$ [baina politikoek (euskaldunak barne) ahalegin guztiak egiten dute, $]_{7}$ elkarrizketa erreal bat edukitzeko? $]_{8}$ 13 [FIL965-2-83-EUS] ${ }^{14}$

After segmentation, we have annotated the main topic of the text or the most important idea of the citizen. This EDU will be the central unit of the RS-tree in the following annotation task ${ }^{15}$. In this

\footnotetext{
${ }^{13}$ [Main Spanish political parties don't want to see us make do well among ourselves. $]_{1}$ [When having dialogue, $]_{2}$ [each should lower her "ambitions", $]_{3}$ [to reach small agreements $]_{4}$ [and, then, arrive at major ones. $]_{5}$ [It is ok that citizens to talk to each other and fora, $]_{6}$ [but politicians (including Basques) do everything they can $]_{7}[\text { to have a real dialogue? }]_{8}$

${ }^{14}$ Text ID-Question-Group-Language.

${ }^{15}$ The Central Unit is considered the correlate in RST to the Central Claim of an argumentation scheme (Peldszus and Stede, 2016) and important for future steps in the annotation of this corpus.
} 
text, we think that the most important sentence of the three is composed of segments 2 to 5 and if we put off all the adverbial clauses of this example, the main EDU is in bold type $\left(\mathrm{EDU}_{3}\right)$.

\subsubsection{Annotation and Evaluation of Rhetorical Relations}

After determining the main topic of the text, as seen in the Example 2, one annotator has labeled the entire corpus, and a part of the corpus was double annotated (A1 and A2) to measure the inter-annotator agreement.

(2) ANTITHESIS (s ("1"), n (INTERPRETATION (n (CIRCUMSTANCE (s ("2"), n (PURPOSE (n (“3”), s (SEQUENCE (n (“4”), n (“5"))))))), s (CONCESSION (s (“6”), n (PURPOSE (n (“7”), s (“8”)))))))) [FIL965-2-83-EUS]

We evaluated the most difficult task of the rhetorical annotation, which is the relation labeling in regards to RST extended and collapsed relation-sets. Results of the qualitative evaluation revealed low $(30 \%)$ and moderate $(46 \%)$ inter-coder agreement subsequently for extended and collapsed relationsets. The comparative analysis showed differences regarding central unit and nuclearity explaining, partially, low agreement. In short, the interpretation was not very different, but annotators formalized trees in different ways.

We tried that to reduce the ambivalence of interpretations harmonizing the macro-structures of RStrees at each stage, and we realized we had to change some decisions taken to build the corpus. Initially, we analyzed texts responding to different aspects of the same question as independent text-units ${ }^{16}$. For example, we differentiated as independent text-units texts underlining 'problems' and 'opportunities' in response to the same question. But taken together we found most texts at this stage were structured with a central claim and a satellite of a SOLUTIONHOOD relation. This approach diminished the ambivalence of interpretation between annotators in regards to central unit detection and, correspondingly,

\footnotetext{
${ }^{16}$ The reason to take that decision was that in the original reports those aspects of citizens' responses were written separately.
}

\begin{tabular}{|l|l|rr|}
\hline TextID & Matches & Recall & Precision \\
\hline FIL102 & 43 of 49 & 0.934 & 0.877 \\
FIL196 & 50 of 51 & 0.980 & 0.980 \\
FIL1264 & 29 of 35 & 0.828 & 0.828 \\
FIL1713 & 56 of 61 & 0.918 & 0.918 \\
FIL2480 & 36 of 43 & 0.947 & 0.837 \\
FIL2517 & 31 of 41 & 0.756 & 0.756 \\
\hline
\end{tabular}

Table 2: Inter-annotator agreement of relation assignment using RSTeval, after a training session and harmonizing segmentation and scope of the rhetorical relations

affected positively regarding the agreement in relation labeling.

After a training session for the novel annotator, we recomposed the corpus following new guidelines and re-annotated it.

Finally, taking harmonized RS-trees based on macro-structural templates as a departure point for the annotation of texts at each stage of discussion, we re-annotated a sample of the original corpus and measured inter-coder agreement using the freely available on-line tool RSTeval (Table 2).

\subsubsection{Text Classification, Cluster relations and Statistical Analysis}

At the end, we were able to classify each text according to $i$ ) stage (1st and 2nd phase and initial (Inform/Propose), middle (Consider) or final stage (Revise)), ii) language (Basque or Spanish) and $i i i$ ) group composition (linguistically heterogeneous or homogeneous) ${ }^{17}$.

To answer our research questions, we classify coherence relations ${ }^{18}$ in classes according to the taxonomy presented by Benamara and Taboada (2015) as follows: $a$ ) Temporal class $\left.{ }^{19} . b\right)$ Structuring class ${ }^{20}$. c) Thematic class is further divided in two sub-

\footnotetext{
$\left.{ }^{17} i\right)$ and $\left.i i\right)$ are relevant aspects of deliberation genre from the perspective of Linguistic Analysis and Argumentation Theory. ii) and $i i i)$ are relevant aspects of deliberation genre from the perspective of Deliberative Democratic Theory (Caluwaerts and Deschouwer, 2014).

${ }^{18}$ This taxonomy also includes more semantically oriented relations as, for example, topic-comment SDRT relations. In this case, we have used it to classify only relations annotated in our corpus.

${ }^{19}$ Only includes SEQUENCE.

${ }^{20}$ Conjunction, Disjunction and List.
} 


\begin{tabular}{|l|r|r|r|r|r|r|r|r|r|r|}
\hline & \multicolumn{4}{|c|}{ Spanish Set } & \multicolumn{4}{|c|}{ Basque Set } \\
\hline Class & \multicolumn{2}{|c|}{ Heter. } & \multicolumn{1}{|c|}{ Homog. } & Total & \multicolumn{2}{|c|}{ Heter. } & \multicolumn{1}{|c|}{ Homog. } & Total \\
\hline Temporal & 7 & $1.1 \%$ & 4 & $0.7 \%$ & $\mathbf{1 1}$ & 8 & $1.1 \%$ & 5 & $0.9 \%$ & $\mathbf{1 3}$ \\
Structuring & 96 & $15.5 \%$ & 95 & $16.2 \%$ & $\mathbf{1 9 1}$ & 112 & $14.8 \%$ & 97 & $18.0 \%$ & $\mathbf{2 0 9}$ \\
Elaboration & 116 & $18.7 \%$ & 110 & $19.7 \%$ & $\mathbf{2 2 6}$ & 157 & $20.7 \%$ & 132 & $24.4 \%$ & $\mathbf{2 8 9}$ \\
Framing & 69 & $11.1 \%$ & 81 & $14.5 \%$ & $\mathbf{1 5 0}$ & 70 & $9.2 \%$ & 41 & $7.6 \%$ & $\mathbf{1 1 1}$ \\
Causal & 105 & $16.9 \%$ & 87 & $15.6 \%$ & $\mathbf{1 9 2}$ & 126 & $16.6 \%$ & 75 & $13.9 \%$ & $\mathbf{2 0 1}$ \\
Purpose & 55 & $8.9 \%$ & 42 & $7.5 \%$ & $\mathbf{9 7}$ & 55 & $7.2 \%$ & 52 & $9.6 \%$ & $\mathbf{1 0 7}$ \\
Support & 105 & $16.9 \%$ & 92 & $16.5 \%$ & $\mathbf{1 9 7}$ & 158 & $20.8 \%$ & 86 & $15.9 \%$ & $\mathbf{2 4 4}$ \\
Opposition & 68 & $11.0 \%$ & 46 & $8.3 \%$ & $\mathbf{1 1 4}$ & 73 & $9.6 \%$ & 52 & $9.6 \%$ & $\mathbf{1 2 5}$ \\
\hline
\end{tabular}

Table 3: Total relations per class by language and group composition.

classes: Elaboration ${ }^{21}$ and Framing ${ }^{22} . d$ ) Argumentative class, divided in two subclasses: Causal ${ }^{23}$ and Argumentative; and the latter further divided in two subclasses: Support ${ }^{24}$ and Opposition ${ }^{2526}$.

Finally, to get the most informative approximation possible we cluster relations at the lower level of the hierarchy of each class and we order classes from less argumentative (Structuring) to more argumentative (Argumentative Opposition) in the light of the 'deliberative minimum, ${ }^{27}$. We test for high correlation among our independent variables excluding multicollinearity, and we make an ordinal logistic regression taking relation class as our dependent variable and stage, group-composition and language as our independent variables.

\section{Results}

In Table 3 we summarize the type and the frequency of each relation per subclass, group composition, and language. At first sight, we could see that, except for texts written in Basque in heterogeneous groups, each column follows the same order from most frequent to less frequent relation class (Argumentative, Thematic, Structuring, and Tempo-

\footnotetext{
${ }^{21}$ Elaboration, Summary, Restatement, Means and we have also included PREPARATION.

${ }^{22}$ BACKGROUND and CIRCUMSTANCE.

${ }^{23}$ CAuse, Result, Purpose, Conditional group and we have also included SOLUTION-HOOD.

${ }^{24}$ Motivation, Evidence, Justify, Evaluation, InTERPRETATION and we also included ENABLEMENT.

${ }^{25}$ CONTRAST, CONCESSION and ANTITHESIS.

${ }^{26}$ JOINT and UNION were excluded from clustering and, therefore, not considered for analysis and interpretation.

${ }^{27} \mathrm{We}$ order them from less to more argumentative as follows: Structuring-Temporal-Elaboration-FramingCausal-Purpose-Argumentative Support-Argumentative Opposition.
}

\begin{tabular}{|l|cc|ccc|}
\hline Phase & \multicolumn{2}{|c|}{ First Phase } & \multicolumn{3}{|c|}{ Second Phase } \\
\hline Class & Prop. & Rev. & Inf. & Cons. & Rev. \\
\hline Non-arg. & $\mathbf{5 6 \%}$ & $\mathbf{6 0 \%}$ & $45 \%$ & $40 \%$ & $45 \%$ \\
Arg. & $44 \%$ & $39 \%$ & $\mathbf{5 4 \%}$ & $\mathbf{5 9 \%}$ & $\mathbf{5 5 \%}$ \\
\hline
\end{tabular}

Table 4: Stages per phase and argumentative vs nonargumentative classes.

\begin{tabular}{|r|rc|c|}
\hline Source & W. Chi-Squ. & Df. & Sig. \\
\hline G. Comp. & 6.245 & 1 & $\mathbf{0 . 0 1 2}$ \\
Stage & 35.090 & 4 & $\mathbf{0 . 0 0 0}$ \\
Language & 0.181 & 1 & 0.670 \\
\hline
\end{tabular}

Table 5: Tests of Model Effects

ral). Within classes, there are more Elaborative relations than Framing relations and more Argumentative than Causal.

In Table 4 we have dichotomized argumentative and non-argumentative classes per phase. Results show that, contrary to our expectations, the balance between argumentative and non-argumentative classes only differs slightly through stages within the same phase. But, it is interesting to see that there are differences if we compare both phases: in the first phase we find more non-argumentative class relations but, in the second phase, argumentative class relations score higher than in the first.

In response to our research questions, the Tests of Model Effects (Table 5) show that the stage of discussion and the composition of the group have a significant effect on the prediction of whether texts will be more argumentative. On the contrary, language has not a statistically significant effect.

To get a more detailed approximation of the directionality and size of these effects, we summarize main results of the ordinal logistic regression in Table 6 .

Our first research question asks whether the lan- 


\begin{tabular}{|r|c|ccc|c|cc|}
\hline & \multicolumn{5}{|c|}{ Hypothesis Test } & & \multicolumn{2}{c|}{ 95\% Wald CI for Exp(B) } \\
\hline Parameter & B. & W. Chi-Sq. & Df. & Sig. & Exp(B) & Lower & Upper \\
\hline Heterog. & 0.178 & 6.245 & 1 & $\mathbf{0 . 0 1 2}$ & 1.195 & 1.039 & 1.374 \\
Homog. & 0 &. &. &. & 1 &. &. \\
\hline Propose (I) & -0.449 & 14.154 & 1 & $\mathbf{0 . 0 0 0}$ & 0.639 & 0.505 & 0.807 \\
Revise (I) & -0.590 & 17.142 & 1 & $\mathbf{0 . 0 0 0}$ & 0.555 & 0.420 & 0.733 \\
Inform (II) & -0.216 & 3.253 & 1 & 0.071 & 0.806 & 0.638 & 1.019 \\
Consider (II) & -0.026 & 0.047 & 1 & 0.828 & 0.974 & 0.771 & 1.232 \\
Revise (II) & 0 &. &. &. & 1 &. &. \\
\hline Spanish & -0.030 & 0.181 & 1 & 0.670 & 0.970 & 0.844 & 1.115 \\
Basque & 0 &. &. &. & 1 &. &. \\
\hline
\end{tabular}

Table 6: Parameter Estimates

guage has an effect on the relational discourse structure of texts. Results show that the odds of texts written in Spanish being more argumentative are almost equal in comparison to those written in Basque. Therefore, language does not have a statistically significant effect on the degree of argumentativeness of texts in our corpus.

Our second research question asks whether the composition of the group has an effect on the relational discourse structure of texts. In this case, results show that the odds of texts being more argumentative are slightly higher (1.195; 95\% CI, 1.039 to 1.374) in linguistically heterogeneous groups than in homogeneous groups. The difference is rather small, but the effect is statistically significant $\left(x^{2}(1)=6.245, p=.012\right)$.

Finally, stages of discussion have an effect on the relational discourse structure of texts in our corpus, but this effect is statistically significant only of both stages in the first phase.

In the second phase, results show that the odds of the relational discourse structure of texts being more argumentative are similar at any stage of discussion. It is slightly lower at the Inform stage, but not significantly. On the contrary, at any stage in the first phase, the odds of the relational discourse structure of texts being more argumentative are lower in comparison to our reference category. This result is especially interesting because the question was the same at both phases, and we used the same macrostructural template.

In this case, the odds of texts in the Revise stage in the first phase being more argumentative are half (.555; 95\% CI, .420 to .733) that of those in the same stage in the second phase, a statistically significant effect, $x^{2}(1)=17.142, p<.001$.

In sum, results show that the stage of discussion and the composition of the group have an effect on the relational discourse structure of texts in our corpus, but not the language. The odds ratios of being more argumentative are higher in the second phase and in linguistically heterogeneous groups. The size of this effect is greater for stages but rather small of group composition. These results may suggest the relational structures of these texts have common patterns associated with their genre. But further analysis is necessary, for example, including other variables or comparison to other genres.

\section{Related Works}

This research builds on previous attempts to analyze argumentation using RST.

Azar (1999) was first to propose RST as an alternative to the so-called "Toulmin Model" (Toul$\min , 1958$ ) for the analysis of argumentation. He used the satellite-nucleus distinction to identify arguments and conclusions for five types of relations: Evidence, Antithesis and Concession (persuader), Justify (justifier), and Motivation (incentive).

More recently, Green (2010; 2015), suggested the "Toulmin model" is more appropriate, but she outlined a proposal (ArgRST) where data and claim of an argument are represented respectively as the satellite and nucleus of an RS-tree.

Texts in our corpus are similar to the 'Postdam Corpus' (Peldszus and Stede, 2016; Stede et al., 2016) composed of 112 argumentative micro-texts written in response to trigger questions aimed at getting argumentatively dense texts. Authors conduct a 
parallel annotation using different methods for the analysis of discourse structures - Segmented Discourse Representation Theory (SDRT) and Rhetorical Structure Theory (RST) - and argumentation schemes (Freeman, 2011).

On the other hand, Hirst et al. (2014) combine linguistically enriched RST parsing based on HILDA discourse parser, and content analysis to analyze argumentation in political speech. They applied their model to the analysis of issue framing and ideological position in historical and contemporary proceedings of British, Canadian and Dutch parliaments.

These studies (Hirst et al., 2014; Stede et al., 2016) share that, despite limitations (Biran and Rambow, 2011), rhetorical structures can be considered appropriate for the analysis of the discourse structure of argumentative texts. But, they invite to the parallel annotation of argumentation schemes and relational discourse structures to enhance their translatability.

Regarding the analysis of argumentation in Basque and Spanish using RST, previous research shows that the nucleus of a rhetorical tree can be seen as the central claim of an argumentation scheme (Iruskieta et al., 2014). Moreover, promising advances have been made regarding automatic segmentation for $\mathrm{Spanish}^{28}$ and Basque ${ }^{29}$ (Iruskieta and Zapirain, 2015), central unit detection ${ }^{30}$ (Bengoetxea et al., 2017) and causal coherence relation annotation in the baseline hierarchical level of the RS-tree (Kortajarena, 2016).

\section{Conclusions and future work}

First, in this paper, we report the creation of a Basque-Spanish bilingual corpus composed by 200 argumentative micro-texts. We have annotated the corpus following usual standards of RST and results are freely available for further analysis in an online database $^{31}$. To our knowledge, this is the first genre

\footnotetext{
${ }^{28}$ The Spanish segmenter DiSeg can be tested online at http://sistema-artext.com/diseg/ (da Cunha et al., 2017).

${ }^{29}$ The segmenter can be tested online at http://ixa2. si.ehu.es/EusEduSeg/EusEduSeg.pl.

${ }^{30}$ The central unit $(\mathrm{CU})$ detector can be tested online at http://ixa2.si.ehu.es/CU-detector/.

${ }^{31}$ The annotated corpus can be consulted online at http: //ixa2.si.ehu.es/diskurtsoa/rstfilo/.
}

analysis of the relational discourse structure of texts applied to deliberative discourse.

Second, the analysis of relation classes has shown that the composition of the group and stages of discussion significantly affect the relational discourse structure of texts. Indeed, texts from groups with participants from different linguistic communities and more controversial questions ruling discussion are closer to the 'deliberative minimum.'

Finally, besides statistically significant differences and given the small size of effects, we could also suggest there are common patterns. Therefore, it is interesting for future research to see whether these patterns are unique and, therefore, genre related or common to other corpora from different genres.

Further steps will follow recommendations regarding parallel annotation of RS-trees and argumentation schemes (Stede et al., 2016). We will also linguistically enrich the annotation signaling Discourse Relational Devices following (2013).

\section{Acknowledgments}

This study was carried out within the framework of the following projects: IXA-CLARIN-K Centre, IXA group, Research Group (GIU16/16) and TUNER (TIN2015-65308-C5-1-R). Science and Society (IT644-13), PRAXIS Research Group.

We would like to thank Esther Miranda and Kike Fernandez (IXA Group) for their help in designing the web page.

\section{References}

[Aduriz et al.2003] Itziar Aduriz, Izaskun Aldezabal, Inaki Alegria, J Arriola, A Díaz de Ilarraza, Nerea Ezeiza, and Koldo Gojenola. 2003. Finite state applications for Basque. In EACL 2003 Workshop on Finite-State Methods in Natural Language Processing, pages 3-11.

[Azar1999] Moshe Azar. 1999. Argumentative text as rhetorical structure: An application of Rhetorical Structure Theory. Argumentation, 13(1):97-114.

[Benamara and Taboada2015] Farah Benamara and Maite Taboada. 2015. Mapping different rhetorical relation annotations: A proposal. In Proceedings of the Fourth Joint Conference on Lexical and Computational Semantics, * SEM, volume 2012, pages 147-152. 
[Bengoetxea et al.2017] Kepa Bengoetxea, Aitziber Atutxa, and Mikel Iruskieta. 2017. Un detector de la unidad central de un texto basado en técnicas de aprendizaje automático en textos científicos para el euskera. Procesamiento del Lenguaje Natural, 58:37-44.

[Bhatia2004] Vijay Bhatia. 2004. Worlds of written discourse: A genre-based view. A\&C Black.

[Biran and Rambow2011] Or Biran and Owen Rambow. 2011. Identifying justifications in written dialogs by classifying text as argumentative. International Journal of Semantic Computing, 5(04):363-381.

[Caluwaerts and Deschouwer2014] Didier Caluwaerts and Kris Deschouwer. 2014. Building bridges across political divides: Experiments on deliberative democracy in deeply divided Belgium. European Political Science Review, 6(03):427-450.

[Carreras et al.2004] Xavier Carreras, Isaac Chao, Lluis Padró, and Muntsa Padró. 2004. Freeling: An opensource suite of language analyzers. In $L R E C$, pages 239-242.

[Collins and Nerlich2015] Luke Collins and Brigitte Nerlich. 2015. Examining user comments for deliberative democracy: A corpus-driven analysis of the climate change debate online. Environmental Communication, 9(2):189-207.

[da Cunha et al.2017] Iria da Cunha, M Amor Montané, and Luis Hysa. 2017. The artext prototype: An automatic system for writing specialized texts. $E A C L$ 2017, pages 57-60.

[Fairclough and Fairclough2013] Isabela Fairclough and Norman Fairclough. 2013. Political discourse analysis: A method for advanced students. Routledge.

[Fairclough2016] Isabela Fairclough. 2016. Deliberative discourse. The Routledge Handbook of Critical Discourse Analysis. London: Routledge.

[Fischer2012] Frank Fischer. 2012. The argumentative turn revisited: public policy as communicative practice. Duke University Press.

[Freeman2011] James B Freeman. 2011. Argument Structure: Representation and Theory. Springer Science \& Business Media.

[Green2010] Nancy L. Green. 2010. Representation of argumentation in text with Rhetorical Structure Theory. Argumentation, 24(2):181-196.

[Green2015] Nancy L Green. 2015. Identifying argumentation schemes in genetics research articles. NAACL HLT 2015, pages 12-21.

[Hirst et al.2014] Graeme Hirst, Vanessa Wei Feng, Christopher Cochrane, and Nona Naderi. 2014. Argumentation, ideology, and issue framing in parliamentary discourse. In ArgNLP.
[Iruskieta and Zapirain2015] Mikel Iruskieta and Benat Zapirain. 2015. Euseduseg: A dependency-based EDU segmentation for Basque. Procesamiento del Lenguaje Natural, 55:41-48.

[Iruskieta et al.2014] Mikel Iruskieta, Arantza Díaz de Ilarraza, and Mikel Lersundi. 2014. The annotation of the central unit in rhetorical structure trees: A key step in annotating rhetorical relations. In COLING, pages 466-475.

[Iruskieta et al.2015a] Mikel Iruskieta, Iria Da Cunha, and Maite Taboada. 2015a. A qualitative comparison method for rhetorical structures: identifying different discourse structures in multilingual corpora. Language resources and evaluation, 49(2):263-309.

[Iruskieta et al.2015b] Mikel Iruskieta, Arantza Diaz de Ilarraza, and Mikel Lersundi. 2015b. Establishing criteria for RST-based discourse segmentation and annotation for texts in Basque. Corpus Linguistics and Linguistic Theory, 11(2):303-334.

[Keating and Bray2006] Michael Keating and Zoe Bray. 2006. Renegotiating sovereignty: Basque nationalism and the rise and fall of the ibarretxe plan. Ethnopolitics, 5(4):347-364.

[Kortajarena2016] Axier Kortajarena. 2016. Koherentziazko diskurtso erlazioen detekzio automatikoa patroien bidez: XMLko erlazio-egiturak oinarri hartuta. HAP master work.

[Mann and Thompson1988] William C. Mann and Sandra A. Thompson. 1988. Rhetorical structure theory: Toward a functional theory of text organization. TextInterdisciplinary Journal for the Study of Discourse, 8(3):243-281.

[Maziero et al.2009] Erick Galani Maziero, Thiago Alexandre Salgueiro Pardo, and Núcleo Interinstitucional de Lingüística Computacional. 2009. Automatização de um método de avaliação de estruturas retóricas. In Proceedings of the RST Brazilian Meeting, pages 1-9.

[McBurney et al.2007] Peter McBurney, David Hitchcock, and Simon Parsons. 2007. The eightfold way of deliberation dialogue. International Journal of Intelligent Systems, 22(1):95-132.

[Miller1984] Carolyn R Miller. 1984. Genre as social action. Quarterly journal of speech, 70(2):151-167.

[Murray et al.2013] Tom Murray, Xiaoxi Xu, and Beverly Park Woolf. 2013. An exploration of text analysis methods to identify social deliberative skill. In International Conference on Artificial Intelligence in Education, pages 811-814. Springer.

[O’Donnell2000] Michael O’Donnell. 2000. RSTTool 2.4: a markup tool for Rhetorical Structure Theory. In Proceedings of the first international conference on Natural language generation-Volume 14, pages 253256. Association for Computational Linguistics. 
[Pardo2005] Thiago Alexandre Salgueiro Pardo. 2005. Métodos para análise discursiva automática. Ph.D. thesis, Instituto de Ciências Matemáticas e de Computação.

[Peldszus and Stede2016] Andreas Peldszus and Manfred Stede. 2016. Rhetorical structure and argumentation structure in monologue text. In Proceedings of the 3rd Workshop on Argument Mining, pages 103-112. ACL.

[Stede et al.2016] Manfred Stede, Stergos Afantenos, Andreas Peldszus, Nicholas Asher, and Jeremy Perret. 2016. Parallel discourse annotations on a corpus of short texts. In Proceedings of the International Conference on Language Resources and Evaluation (LREC), Portoroz, pages 1051-1058.

[Steiner2012] Jürg Steiner. 2012. The foundations of deliberative democracy: Empirical research and normative implications. Cambridge University Press.

[Swales1990] John Swales. 1990. Genre analysis: English in academic and research settings. Cambridge University Press.

[Taboada and Das2013] Maite Taboada and Debopam Das. 2013. Annotation upon annotation: Adding signalling information to a corpus of discourse relations. $D \& D, 4(2): 249-281$.

[Taboada and Gómez-González2012] Maite Taboada and María de los Ángeles Gómez-González. 2012. Discourse markers and coherence relations: Comparison across markers, languages and modalities. Linguistics and the Human Sciences, 6(1-3):17-41.

[Taboada2004] Maite Taboada. 2004. Building coherence and cohesion: Task-oriented dialogue in English and Spanish, volume 129. John Benjamins Publishing.

[Toulmin1958] Stephen Toulmin. 1958. The uses of argument. Cambridge, UK.

[van Eemeren2013] Frans H van Eemeren. 2013. Strategic maneuvering in argumentative discourse in political deliberation. Journal of Argumentation in Context, 2(1):10-31.

[Van Eemeren2016] Frans H. Van Eemeren. 2016. Identifying argumentative patterns: A vital step in the development of pragma-dialectics. Argumentation, 30(1):1-23.

[Walton et al.2014] Douglas Walton, Alice Toniolo, and Timothy J. Norman. 2014. Missing phases of deliberation dialogue for real applications. In Proceedings of the 11th International Workshop on Argumentation in Multi-Agent Systems, pages 1-20. 